\title{
Developing nursing clinical data models addressing delusion and hallucination with Meleis transitions theory as the theoretical reference model: A focus group study
}

\author{
Patrícia Daniela Barata Gonçalves RMSN, RMN, MSc, PhD student ${ }^{1,2,3,4}$ (]) | \\ Carlos Alberto da Cruz Sequeira RMN, MSc, PhD ${ }^{3,4}$ \\ Maria Antónia Taveira da Cruz Paiva e Silva RMSN, MSc, PhD $^{3}$ \\ Abel Avelino de de Paiva e Silva RMSN, MSc, PhD ${ }^{3}$
}

${ }^{1}$ Instituto de Ciências Biomédicas Abel Salazar, Universidade do Porto,

Porto, Portugal

${ }^{2} \mathrm{~B} 3$, Hospital de Magalhães Lemos,

Porto, Portugal

${ }^{3}$ UNIESEP, Escola Superior de Enfermagem do Porto, Porto, Portugal

${ }^{4}$ NursID, Centro de Investigação em

Tecnologias e Serviços de Saúde,

Porto, Portugal

\section{Correspondence}

Patrícia Daniela Barata Gonçalves, Rua Dr. António Bernardino de Almeida, 830, 844, 856, 4200-072 Porto, Portugal.

Email: patriciagoncalves7s@gmail.com

\begin{abstract}
Purpose: To build the final clinical data models regarding the nursing focuses "Delusion" and "Hallucination" with Meleis transitions theory as the theoretical reference model.

Design and Methods: A qualitative descriptive design was adopted, using two focus groups.

Findings: The clinical data models obtained by this study recognize a perspective centered on the client's transition, which emphasizes the role of nursing in relation to a client suffering from delusion and hallucination.

Implications for Nursing Practice: These clinical data models may contribute towards improving nursing clinical decision-making and nursing care quality regarding these areas.
\end{abstract}

KEYWORDS

decision making, delusion, diagnosis, hallucination, intervention, psychiatric nursing

\section{1 | INTRODUCTION}

The development of a nursing clinical data model allows the systematization of the connections between the elements of the nursing process-data, diagnoses and interventions-for a given nursing focus. Clinical data models enable knowledge representation by the experts of a specific area, ${ }^{1}$ in this case, by nurses, and allow structuring all the information related to a given concept. ${ }^{2}$ They provide evidence-based data elements related to nursing care, which may contribute to information exchange, clinical decision support, quality reporting, research, and the improvement of nursing care quality. ${ }^{3}$

Documentation is essential for patient safety and quality of care. ${ }^{4}$ Portugal is at the forefront with regard to the use of health technologies in Europe ${ }^{4,5}$ Nursing progressed from an executive logic-based method to an approach centered on care planning and decision-making. The way care is documented has also evolved in this direction, so the emphasis initially devoted to the mere production of documentary evidence of the actions carried out by nurses has been transferred to the necessary information for sustained and effective clinical decision-making. De Groot et al. ${ }^{6}$ highlight the importance of aligning the nursing records with the steps of the nursing process and Hardiker et al. ${ }^{7}$ advocate that, to improve care processes and subsequent patient outcomes, electronic health records should become decision support systems, providing health professionals guidance when a decision is being taken. Thus, the development and incorporation of clinical data models that represent the "core" concepts of the Nursing discipline, organized and structured based on the available formal knowledge, specifically in the area of mental health and psychiatry, will support nurses in making better decisions, more congruent with the client specific needs. 


\section{1 | Background}

${ }^{1}$ Gonçalves et al. ${ }^{8}$ and ${ }^{2}$ Gonçalves et al. ${ }^{9}$ developed scoping reviews which aimed to identify relevant data, diagnoses and interventions related to the nursing focuses "Delusion" and "Hallucination." This study aims to explore and obtain contributions regarding the data, diagnoses and interventions related to "Delusion" and "Hallucination," identified in these previous scoping reviews and, hence, to reach the final clinical data models addressing these important nursing focuses. Delusion has been defined by the International Council of Nurses (ICN) as an impaired belief: a false sense of reality that cannot be corrected by reason, argument, or persuasion or by evidence of one's own senses. ${ }^{10}$ Hallucination, on the other hand, has been defined as an apparent registration of sensory stimuli which is not actually present, classified according to the senses, such as auditory, visual, olfactory, gustatory or tactile hallucination. ${ }^{10}$

According to Meleis transitions theory, nursing focuses on facilitating the individual's transition process. ${ }^{11,12}$ Throughout life, people continuously experience changes and transitions, which lead to the development of new relationships, new behaviors and new perceptions about themselves. The diagnoses "Delusion" and/or "Hallucination" may lead to experiencing a transition, requiring the individual to implement a set of strategies that support the adaptation to the new condition. The Portuguese Nursing Council ${ }^{13}$ states that the mental health nurse implements individual, family or group psychotherapeutic and sociotherapeutic interventions focused on human responses to health/mental illness and to transitions. It is with this vision in mind that Meleis transitions theory is seen as the conceptual framework/theoretical reference model of this study. Afaf Meleis transitions theory is a scientifically tested middle-range theory, with practical adaptation and applicability and it is a reference model compatible with the legal regulations of nursing in Portugal.

\subsection{Aim}

This study aims to explore and obtain contributions regarding the data, diagnoses and interventions related to "Delusion" and "Hallucination," identified in two scoping reviews performed beforehand and to build the final clinical data models related to the nursing focuses "Delusion" and "Hallucination."

\section{2 | METHODS}

The area of clinical data models has assumed particular importance in Portugal, in the Oporto Nursing School (Escola Superior de Enfermagem do Porto), which formed the Nursing Informatics Systems Research \& Development Center (Centro de Investigacão e Desenvolvimento dos Sistemas de Informação em EnfermagemCIDESI), accredited by the ICN in 2010. Within CIDESI, a project was started, currently called "NursingOntos-Nursing Ontology," which aims to develop an ontology in nursing and clinical data modelsarchetypes-that can be executed on the nursing information systems backend. There is already a joint task force between CIDESI and the Portuguese Nursing Council to fulfill this purpose. ${ }^{14}$ The present study arose in the context of this project.

A detailed clinical model is a logical model designed to express one or more clinical concepts and their context in a standardized and reusable manner, specifying the requirements for clinical information as a discrete set of logical clinical data elements. ${ }^{2}$ Clinical data models may represent the formalization of the disciplinary knowledge in Nursing, by specifying the relevant data for diagnostic reasoning, the diagnoses, the interventions, as well as the epistemological basis of formal knowledge that regulates the relationship between these elements.

To achieve a nursing clinical data model regarding a particular nursing focus, the research works included in the NursingOntos project comprise three phases: (1) content analysis of the diagnoses and interventions regarding the nursing focus integrated in the national parameterization of the nursing information system in use in the Portuguese National Health Service; and focus group study, to obtain contributions on the content analysis performed; (2) scoping review, with the aim of mapping the available scientific evidence regarding the relevant data, diagnoses and interventions related to the selected nursing focus; (3) focus group study, to obtain contributions regarding the data, diagnoses and interventions identified in the scoping review previously performed.

The present study represents the final step of the process of developing nursing clinical data models regarding the nursing focuses "Delusion" and "Hallucination." As mentioned before, the results of two previous scoping reviews constitute the basis of the this study. In Table 1, we present the main results of the scoping review addressing "Delusion"-Data, diagnoses, and interventions addressing the nursing focus "Delusion." In Table 2, we present the main results of the scoping review addressing "Hallucination"Data, diagnoses and interventions addressing the nursing focus "Hallucination."

\subsection{Focus groups}

Two focus groups were formed to explore and obtain contributions regarding the data, diagnoses and interventions related to "Delusion" and "Hallucination" (presented in Tables 1 and 2). A purposive sample of nurses for each focus group was contacted by email. The first focus group had the participation of eleven nursing professionals, specialists in the field of the representation of nursing knowledge, Nursing Information Systems, Ontology, and Clinical data models in Nursing-the NursingOntos focus group. The second was attended by eight nursing professionals who are specialists in Mental health and psychiatric nursing-the Mental Health Specialists Nurses focus group. The authors of this study considered that contributions from both these areas (the representation of nursing knowledge and mental health nursing) would be crucial for the development of the clinical data models regarding "Delusion" and 
TABLE 1 Delusion' scoping review main results (data, diagnoses, and interventions addressing delusion), ${ }^{1}$ Gonçalves et al. ${ }^{8}$

Data Diagnoses Interventions

Data that lead to the diagnosis:

- Beliefs or ideas that cannot be changed through logic

Delusion

- Cognitive behavioral therapy and reason, maintained despite evidence of the contrary

- Unshakeable false beliefs

Diagnostic hypotheses:

- Lack of delusion awareness

Data that characterize the diagnosis:

- Delusion' impact on thinking, behavior, feelings and social relations

- Delusion conviction

- Delusion systematization

- Delusion persistence/duration

- Delusion insight

- Preoccupation associated with delusion

- Delusion fixity/crystallization

- Anguish associated with delusion

TABLE 2 Hallucination' scoping review main results (data, diagnoses, and interventions addressing hallucination), ${ }^{2}$ Gonçalves et al. ${ }^{9}$
- Lack of knowledge about delusion
- Metacognitive training

- Psychoeducation

- Cognitive remediation treatment

- Dialectical behavior therapy

- Occupational therapy delivered by nurses

- Organized program of music therapy, art therapy, cognitive activities, orientation training and exercise

Data Diagnoses Interventions

Data that lead to the diagnosis:

- Perceptions which are not generated by external stimuli

Data that characterize the diagnosis:

- Frequency/duration

- Negative content associated with hallucination

- Intensity

- Positive content associated with hallucination

- Hallucination' impact on thinking, behavior, feelings and social relationships;

- Anguish/distress associated with hallucination

- Hallucination control

- Hallucination awareness

- Positive and negative beliefs/attitudes

towards hallucination
Hallucination

Diagnostic hypotheses:

- Lack of hallucination awareness

- Low hallucination self-control

- Lack of kowledge about hallucination
- Psychoeducation

- Coping strategies/self-control strategies/selfmanagement strategies towards hallucination

- 10-session behavioral management of persistent auditory hallucinations course

- Cognitive behavioral therapy

- Distraction technique

- Cognitive therapy

- Counseling

- Narrative therapy

- Supportive therapy
"Hallucination." The focus groups were conducted in accordance with the methodological guidelines of Krueger and Casey. ${ }^{15}$ Consolidated criteria for reporting qualitative studies steered the designing and reporting of this study. ${ }^{16}$
The following inclusion criteria were applied to constitute the NursingOntos group: (1) Masters or PhD in nursing; (2) member of the Nursing Informatics Systems Research \& Development Center group (CIDESI group, accredited by the ICN); 
(3) prior training in the field of nursing care design or classified nursing language; (4) present or past participation in research projects in the field of nursing care design or classified nursing language. The following inclusion criteria were applied to constitute the Mental Health Specialists Nurses group: (1) Masters or PhD in nursing; (2) nurses specialized in mental health and psychiatric nursing working in this area or professors specialized in mental health and psychiatric nursing teaching in this area.

The focus group meetings (180-min sessions) included a leader (PDBG) who hosted the meeting, explained its aim, encouraged the exchange of ideas and conducted the registration and observation of the group dynamics. A "questioning route" composed of predetermined and sequenced questions was followed. The focus group questions were as follows: (1) "Should any other data, diagnosis or intervention be added to the clinical data models?" (2) "Should any of the data, diagnoses or interventions presented in the "Delusion" clinical data model be added to the "Hallucination" clinical data model or vice versa?" (3) "Should any of the data, diagnoses or interventions presented in the clinical data models be excluded? If so, why?" (4) Is there any other contribution you would like to make with regard to the data, diagnoses and interventions related to "Delusion" and "Hallucination" clinical data models?

For the purposes of qualitative analysis, discussions were recorded in an audio file after obtaining informed consent from all the participants. Data analysis was performed immediately after the focus groups meetings until data saturation was obtained (after two meetings in the NursingOntos focus group and after one meeting in the Mental Health Specialists Nurses focus group). The data was analyzed thematically after transcription of the audiotapes. Transcripts of the audiotapes kept individuals' anonymity, which prevented the researcher to identify the author of each comment. Results from the focus groups were coded by two researchers. No software was used for data analysis.

\subsection{Ethical considerations}

We ensured the confidentiality of all participants and informed that they could at any moment withdraw from the study. Since all participants gave their formal consent, we proceeded with the research. This study was approved by the Scientific Technical Council of the Oporto Nursing School, (no. 25/2016 of 06/27), which encompassed the role of Ethics Committee, in accordance with the principles of the Declaration of Helsinki and subsequent revisions. $^{17}$

\section{FINDINGS}

In the focus group meetings, the following criteria were identified and later applied to the clinical data models:

\subsection{Criteria that emerged from the NursingOntos focus group}

$\checkmark$ The nursing diagnoses "Delusion" and "Hallucination" should only be identified if there is an impairment of client functionality associated with delusion and/or hallucination (if there is not, then no problem exists, so there is no nursing diagnosis, because the client is adapted to these conditions);

$\checkmark$ Considering that Meleis transitions theory constitutes the conceptual framework/theoretical reference model of this study, the clinical data models should embody the principles of this reference model.

- From a perspective in which the focus is on the transition of the client, a view geared towards an opportunity to develop clients' potential based on their personal resources should be adopted, as opposed to making reference to their limitations or deficits. Example: The diagnosis "Lack of knowledge about delusion" was converted into "Potential for improving knowledge about delusion."

- Two domains of interventions should be distinguished: (1) The interventions in which the nurse acts directly on the problem (Example: Performing distraction technique); (2) The interventions in which the nurse interferes in the problem only indirectly and through the client (Example: Teaching the client about delusion)Perspective of the client's transition.

- Meleis transition theory allows approaching delusion and hallucination from the perspective of the client's transition. Hence, also the interventions should embody the principles of this reference model, both in content and in semantics.

If we, nurses, were able to transform data, diagnoses, and interventions into what is intrinsic to us, restructure them according to our own perspective and systematize our professional action in that sense, patients would profit a lot and we would have a great contribution for the society. (NursingOntos focus group, Expert 1).

- According to Meleis transition theory, self-control is always a consequence, an indicator of mastery, therefore, it is not a diagnosis, but an outcome (self-control is a behavior; the nurse interferes in the process of acquiring mastery, and not in the behavior that will be observed when mastery is acquired).

$\checkmark$ For an intervention to be formulated, an action verb is required in the beginning of the sentence, thus, interventions should be reformulated to fulfill this criterion. Example: The intervention consisting in "Metacognitive Training" was converted into "Performing metacognitive training."

$\checkmark$ There must be a univocal relationship between diagnosis and intervention (we cannot have a set of diagnoses for a set of interventions);

$\checkmark$ All contents should be written in accordance to the International Classification for Nursing Practice.

Criteria that emerged from the Mental Health Specialists Nurses focus group:

$\checkmark$ Referential integrity between data, diagnoses and interventions should be achieved, so any diagnosis should be based on sustained 
data and any intervention should respond to and have potential to change the respective nursing diagnosis (*Referential integrity corresponds to the adequate and meaningful relationship between data from different information fields ${ }^{18}$ ). Example: An intervention that consists in "Emotional support" responds to an emotional problem, such as "Anguish associated with delusion/hallucination" (and not to "Delusion" or "Hallucination," because it does not have potential to change delusion or hallucination). For emotional support to be prescribed, an emotional problem must be identified.

$\checkmark$ Any redundant data (which does not add any further information) should be excluded. Example: The data that lead to the diagnosis "Delusion" "Unshakeable beliefs" was removed, because the data "Beliefs or ideas that cannot be changed through logic and reason, maintained despite evidence of the contrary" already provides that information.

$\checkmark$ In addition to characterizing the impact of Delusion and Hallucination on thinking, behavior, feelings and social relations, the impact of Delusion and Hallucination on daily life activities should be characterized;

$\checkmark$ "Delusional content" should be integrated into the data that characterizes "Delusion" (thus, the diagnostic hypotheses regarding Delusion and Hallucination subtypes will be integrated into the data that characterizes the diagnosis);

$\checkmark$ The domains "Awareness of the relationship between non-adherence to the medication regime and delusion/hallucination," "Awareness of the relationship between drug abuse and delusion/hallucination," "Awareness of the relationship between alcohol abuse and delusion/ hallucination," and "Awareness of the relationship between the absence of occupation and delusion/hallucination" should exist in both clinical data models;

$\checkmark$ Interventions that do not represent nursing interventions or whose performance requires specific training (not included in Nursing Specialties) or that are not part of the nurses' autonomous professional practice should be excluded. Example: The intervention "Occupational therapy" was excluded.

$\checkmark$ From a perspective in which the focus is not on the transition of the client, the interventions "Optimizing the environment to decrease sensory stimuli" and "Implementing distraction technique" should be prescribed. "Administering medication" is also an important intervention in this domain, however, it is not part of the nurses' autonomous professional practice.

$\checkmark$ When teaching about delusion or hallucination, the following contents should be specified:

- Teaching about: Delusion/hallucination and the mental disorder which is the basis of delusion/hallucination; delusion/hallucination manifestations; medication regime-indications (action of medication on delusion/on hallucination), medication benefits, side effects of medication and strategies to deal with them; Importance of adopting healthy lifestyle habits and avoiding risky behaviors: importance of sleep, importance of not consuming alcohol or drugs, among others; Delusion/Hallucination prevention/reduction strategies; Warning signs of a possible relapse; Resources available in the community. $\checkmark$ The intervention "Performing cognitive restructuring" should be added to the nursing clinical data model related to "Delusion" (in the approach focused on the perspective of the client's transition).

After the focus groups meetings, the criteria referred above were applied to the data, the diagnoses and the interventions related to "Delusion" and "Hallucination." Through this process, the final clinical data models regarding "Delusion" and "Hallucination" were obtained. They are presented in Figures 1 and 2. Table 3 shows the contents of the 10-session behavioral management of persistent auditory hallucinations course, an important intervention identified in the hallucination clinical data model.

\section{4 | DISCUSSION}

This study allowed the completion of the clinical data models related to the nursing focuses 'Delusion' and "Hallucination." The scoping reviews previously performed made it possible to map the concepts related to the data, diagnoses and interventions in the areas of "Delusion" and "Hallucination." The criteria established by the focus groups and the theoretical framework that supports this study made it possible to "filter" these concepts and establish the relationships between them, allowing the unique role of nurses towards a client suffering from "Delusion" or "Hallucination" to be envisioned. ${ }^{21}$ emphasizes that any profession that does not have discipline specific language to give voice to its role in society, becomes, and remains invisible; indeed it may not exist. According to Meleis, nursing focuses on facilitating the individual's transition process, thus, the role of nurses consists in helping the client to adapt to a new condition and helping them live with it. Bearing this theory in mind, we should consider two different approaches to the client, according to their potential to improve awareness about the problem, knowledge about the problem and, hence, the ability to cope with, manage and control the problem: (1) Approach focused on the perspective of signs and symptoms management and the prevention of complications, and (2) approach focused on the perspective of the client's transition.

In the first approach, the client does not have the potential to improve their awareness or their knowledge of the problem and, hence, the ability to cope with, manage and control it. Therefore, the diagnoses will be centered on the problem itself ("Delusion" and "Hallucination") and the intervention will rely on the nurse's direct action. The nurse will act on the problem and it is the nurse's intervention that will directly influence it.

The second approach, centered on the client's transition, is based on the principles of the Meleis Transitions Theory and represents the greatest contribution of these clinical data models. In this approach, the diagnoses will be centered on the client's potential and will reflect a group of skills they should develop To manage delusion and hallucination. The intervention will rely on the client's action (the client himself will act on the problem) and the nurse's role will consist in assisting them to do something, making them aware of something, teaching them something ... 


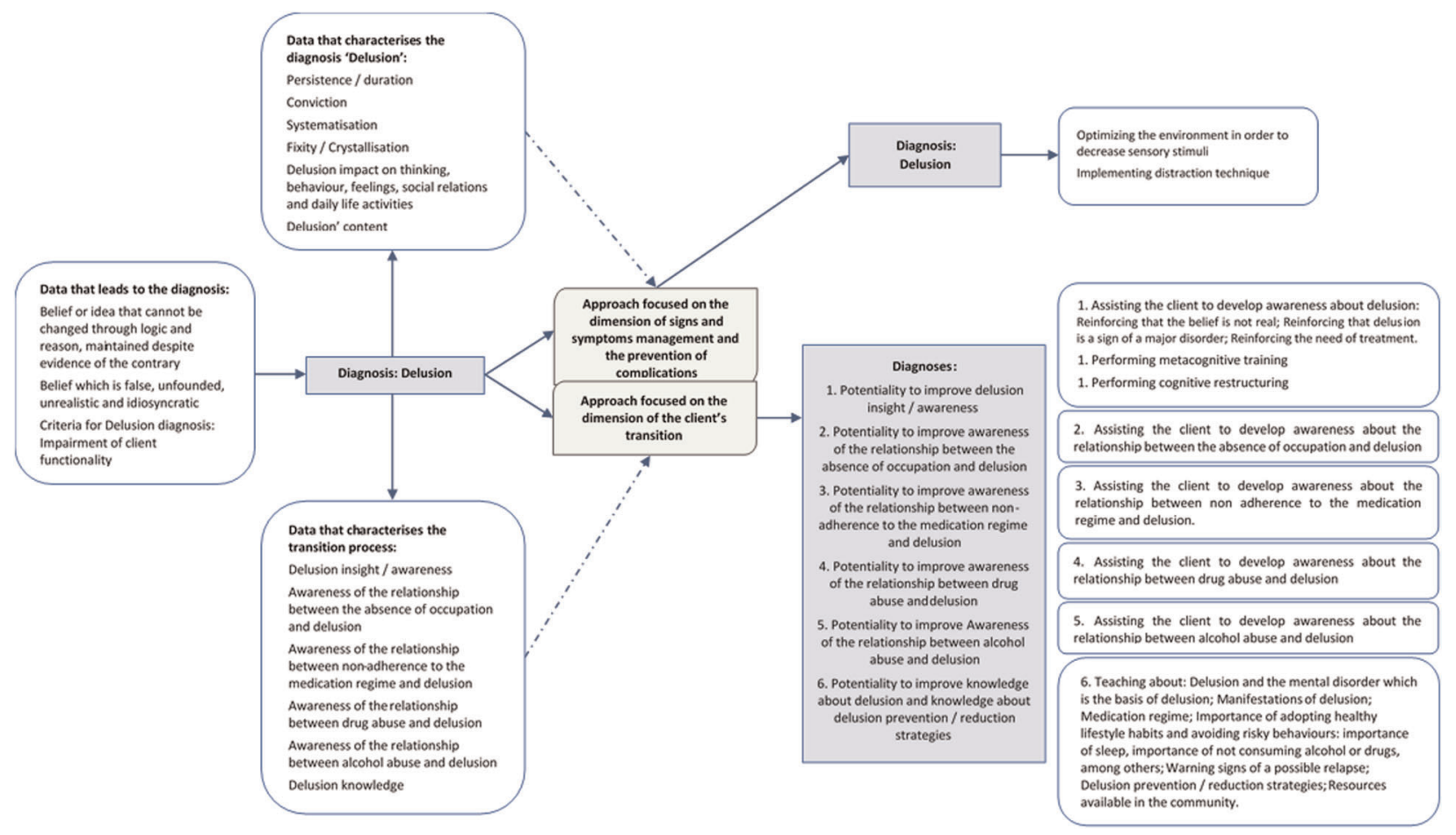

FIGURE 1 Delusion clinical data model [Color figure can be viewed at wileyonlinelibrary.com]

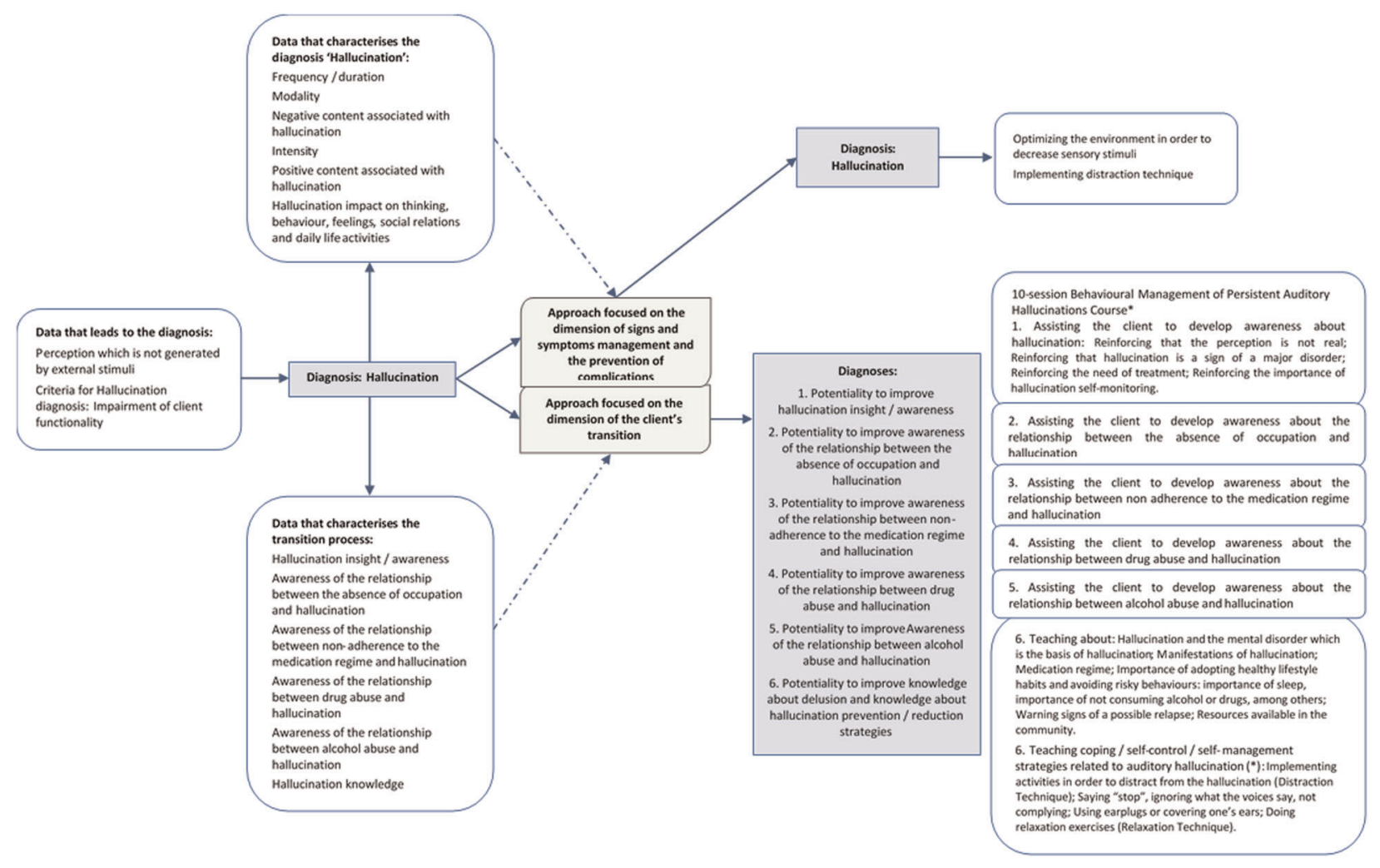

FIGURE 2 Hallucination clinical data model [Color figure can be viewed at wileyonlinelibrary.com] 
TABLE 3 Contents of 10-session behavioral management of persistent auditory hallucinations course

*10-session behavioral management of persistent auditory hallucinations course

(Buffum et al. 2009; Buccheri et al. 2013) 18,19

Intervention:

10 weekly 60' sessions:

$\checkmark$ Sharing the auditory hallucination experiences with the group;

$\checkmark$ Teaching management strategies to the group;

$\checkmark$ Practicing these strategies.

$\checkmark$ Strategies:

(1) Developing symptom awareness;

(2) Hallucination self-monitoring;

(3) Distraction technique:

Doing other things;

Keeping busy with an enjoyable activity and/or helping others;

Talking with someone;

Reading, listening to music, watching television.

(4) Saying "stop," ignoring what the voices say to do, not complying;

(5) Using earplugs or covering one's ears;

(6) Doing relaxation exercises such as taking deep breaths, relaxing the muscles or listening to relaxing music;

(7) Using prescribed medication;

(8) Not using drugs or alcohol.

helping them to develop the skills to cope with/manage/control delusion and hallucination to facilitate their transition.

As the Portuguese Nursing Council ${ }^{13}$ points out, mental health nursing focuses on health promotion, prevention, diagnosis, and intervention towards human responses that are not adapted to the transition processes and which generate suffering. The diagnosis of any mental condition may correspond to one of these responses, triggering the experience of a health-disease transition, in which the nurse plays a crucial role. The changes resulting from mental illness, which may appear abruptly or gradually, generate instability and vulnerability and professional nursing action is expected to support the individual in acquiring the skills that allow them to achieve stability again. It is in this sense that Transition is assumed as one of the central concepts of the nursing discipline. ${ }^{11,12}$

To facilitate the client's transition process, nurses should, first of all, evaluate the properties inherent to the transition. One important property of the transition is awareness, which refers to the person's perception and recognition of a changing situation with specific repercussions. The level of awareness is often reflected in the degree of congruence between the knowledge that the person has about their condition and the limitations that it imposes on their daily activities. ${ }^{11}$
Awareness is crucial in the transition process. If the person is not aware, not recognizing and internalizing the changes that a health problem implies in their life, they will not recognize the need and the importance of the treatment.

In fact, one of the most challenging aspects in psychotic symptoms management is the fact that most clients have limited awareness (insight) of their problem. ${ }^{4,22}$ It is therefore imperative to reinforce delusion/ hallucination awareness. The client will only be able to manage their psychotic symptoms when they develop awareness about them. Metacognitive training for delusion, for example, has proven efficient in improving client awareness about delusion as well as reducing delusion itself. This new treatment modality aims to improve the client's thinking process through the development of their metacognitive skills and insight (awareness). ${ }^{23}$ Also with regard to hallucination, one important strategy related to auditory hallucination management is "developing symptom awareness." $^{20}$

Preparation and knowledge are also essential for a healthy transition. As pointed out by Meleis et al., ${ }^{11}$ the ease or difficulty of the adaptation process will depend on the balance between the requirements associated with the transition and the personal resources. One of the resources that facilitate a healthy transition is the information acquired through health professionals. ${ }^{11} \mathrm{Ma}$ et al. ${ }^{24}$ advocate that mental health and psychiatric professionals should develop intervention programs for clients with auditory hallucination that include promoting clients' knowledge about their voices, teaching them to accept auditory hallucination as a symptom, encouraging them to treat the content of the voices with indifference and developing their coping strategies to deal with this phenomenon.

In fact, awareness and knowledge are fundamental requirements for delusion and hallucination self-management/self-control/coping, which are crucial goals for nurses assisiting the client in their transition process. A client cannot achieve self-control in relation to delusion or hallucination if they are not aware of delusion or hallucination or if they do not have the essential knowledge about delusion or hallucination. Self-control is a consequence and is related to the acquisition of mastery. ${ }^{11}$ According to Meleis et al., ${ }^{11}$ one important process indicator in the transition domain, which indicates whether the person is on the path to health or towards greater vulnerability, is the development of trust and the ability to cope. Psychotic symptoms are based on cognitive biases that trigger, worsen or maintain them. Metacognitive training, for example, aims to make clients aware of these cognitive biases, train them to critically detect these distortions and help them improve their problem-solving repertoire and their delusion management abilities. Education about coping strategies is provided to the client. ${ }^{25}$

Auditory hallucination self-control may be considered one of its fundamental domains. Chadwick and Hemingway, ${ }^{26}$ in their narrative literature review with the aim of analyzing the effectiveness of interventions focused on understanding the experience of hearing voices verified that none of the analyzed articles sought to alter or eliminate the auditory hallucination. Instead, they focused on issues, such as "learning to live with the voices," "accepting the voices and improving the relationship with them" and "reducing the stress associated with auditory hallucination." Thus, although the auditory hallucination remains, its 
presence is no longer a problem. This study highlights the importance of interventions that assist the client in understanding and controlling their psychotic symptoms, as well as exploring the hallucinations content and meaning. In the 1990s, the School of Nursing and Health Professions, University of California, San Francisco, developed symptom management strategies to help clients dealing with their symptoms, combining these strategies with behavioral approaches implemented in groups (Buccheri et al. 1996 as cited by ${ }^{27}$ ). A quasi-experimental study performed by Yang et al. ${ }^{27}$ concluded that the implementation of the Auditory Hallucination Symptom Management Course appears to be effective in improving hallucination self-control.

Finally, although familiar and societal conditions are not addressed in the clinical data models developed, it is important to highlight that these resources may also facilitate or inhibit a healthy transition. Meleis et al. ${ }^{11}$ advocate that, for example, support from partners and families are facilitators for a healthy transition and that viewing a transitional event as stigmatized and with stereotyped meanings tends to interfere in the process of healthy transition.

\section{1 | Limitations}

Despite the interesting results obtained by this study, it does bear some limitations. The fact that the focus group with specialists in mental health nursing only met during one session, despite allowing data saturation, did not allow potential variations in the opinion of the experts over time to be perceived. Besides, the lack of an external observer in the focus groups corresponds to a limitation because this did not allow us to obtain data centered on the nonverbal communication of the participants nor on the subliminal messages. The fact that both focus groups were constituted by Portuguese nurses only may represent a limitation, because it did not allow perspectives from different countries or cultures to be considered. Finally, the fact that Meleis Transitions Theory represents the theoretical reference model of this study can be seen both as a strength and as a limitation; a strength because it provides this study with a theoretical reference guide that supports the results, a limitation because using a theoretical framework as a reference may limit the vision through which the results are perceived.

\section{5 | CONCLUSION}

The clinical data models obtained by this study contribute to the systematization of the connections between the elements of the nursing process-data, diagnoses and interventions-for the nursing focuses "Delusion" and "Hallucination," constituting clinical guides for care planning and decision-making by Portuguese nurses regarding these nursing focuses. These clinical data models present some of the "core" concepts of "Delusion" and "Hallucination," in a nursing perspective, organized and structured based on the available formal knowledge and they underpin the role of Nursing in relation to a client suffering from these conditions.

\section{6 | IMPLICATIONS FOR PRACTICE}

Clinical data models provide evidence-based data elements related to nursing care, which may contribute to information exchange, clinical decision support, quality reporting, research, and the improvement of nursing care quality. ${ }^{3}$ Interoperability refers to the ability of different information systems, devices and applications to access, exchange, integrate, and cooperatively use data in a coordinated manner, within and across organizational, regional and national boundaries. Interoperability is important to provide timely and seamless portability of information, optimize the health of individuals and populations and reduce healthcare costs. $^{28,29}$

The application of the clinical data models developed by this study may contribute to the improvement of nursing information systems and semantic interoperability and, therefore, to the production of nursingsensitive indicators within the areas of "Delusion" and "Hallucination" in Portugal. Furthermore, the existence of clinical data models should help enhance clinical practices by supporting decision-making processes by Portuguese nurses and improving their clinical reasoning and critical thinking towards the application of the nursing process within the areas of "Delusion" and "Hallucination."

This study may be relevant in international healthcare, because it provides information to other countries about the process behind nursing clinical data models creation, which may encourage the replication of the process in different international contexts.

We recommend that further research should be carried out to test the applicability of the clinical data models developed in clinical practice and to develop clinical data models addressing other nursing focuses.

\section{ACKNOWLEDGMENT}

This study received no specific grant from any funding agency in the public, commercial, or not-for-profit sectors.

\section{CONFLICT OF INTERESTS}

The authors declared no potential conflict of interests with respect to the research, authorship, and/or publication of this article.

\section{AUTHOR CONTRIBUTION}

Patrícia Daniela Barata Gonçalves, Carlos Alberto da Cruz Sequeira, Maria Antónia Taveira da Cruz Paiva e Silva, and Abel Avelino de Paiva e Silva completed the study design and critical revisions for important intellectual content. Patrícia Daniela Barata Gonçalves completed data collection. Patrícia Daniela Barata Gonçalves and Carlos Alberto da Cruz Sequeira completed data analysis. Carlos Alberto da Cruz Sequeira and Maria Antónia Taveira da Cruz Paiva e Silva completed the study supervision. Patrícia Daniela Barata Gonçalves completed manuscript writing. All authors listed meet the authorship criteria according to the latest guidelines of the International Committee of Medical Journal Editors. All authors are in agreement with the manuscript.

\section{DATA AVAILABILITY STATEMENT}

Author elects to not share data. 


\section{ETHICS STATEMENT}

This study was approved by the Scientific Technical Council of the Oporto Nursing School, (no. 25/2016 of 06/27), which encompassed the role of Ethics Committee.

\section{ORCID}

Patrícia Daniela Barata Gonçalves (D) https://orcid.org/0000-00026329-3897

Carlos Alberto da Cruz Sequeira (D) https://orcid.org/0000-0002 5620-3478

\section{REFERENCES}

1. Spigolon D, Moro C. Essential data set's archetypes for nursing care of endometriosis clients. Revista Gaúcha de Enfermagem. 2012;33(4):22-32. https://doi.org/10.1590/S1983-14472012000400003

2. International Organization for Standardization. Health informatics: Detailed clinical models, characteristics and processes. International Organization for Standardization. ISO/TS 13972. International Organization for Standardization; 2015. https://www.iso.org/obp/ui/\#iso:std:iso: ts:13972:ed-1:v1:en. Accessed April 2021.

3. Chow $M$, Beene $M, O$ 'Brien $A$, et al. A nursing information model process for interoperability. J Am Med Inform Assoc. 2015;22(3): 608-614. https://doi.org/10.1093/jamia/ocu026

4. Nursing and Midwifery Council. The Code. Professional Standards of Practice and Behaviour for Nurses, Midwifes and Nursing Associates. Nursing \& Midwifery Council. 2018.

5. European Observatory on Health Systems and Policies. Health System Review: Portugal, Final Report. World Health Organization; 2018.

6. De Groot K, Triemstra M, Paans W, Francke A. Quality criteria, instruments, and requirements for nursing documentation: a systematic review of systematic reviews. J Adv Nurs. 2019;75(7): 1379-1393. https://doi.org/10.1111/jan.13919

7. Hardiker N, Dowding D, Dykes P, Sermeus W. Reinterpreting the nursing record for an electronic context. Int J Med Inform. 2019;127: 120-126. https://doi.org/10.1016/j.ijmedinf.2019.04.021

8. ${ }^{1}$ Gonçalves P, Sampaio F, Sequeira C, Silva M. Data, diagnoses, and interventions addressing the nursing focus "delusion": a scoping review. Perspect Psychiatr Care. 2019;56(1):175-187. https://doi.org/ 10.1111/ppc.12401

9. ${ }^{2}$ Gonçalves $P$, Sampaio F, Sequeira C, Silva M. Nursing process addressing the nursing focus "Hallucination": a scoping review. Clin Nurs Res. 2019;30:1-19. https://doi.org/10.1177/1054773819877534

10. International Council of Nurses. ICNP ${ }^{\circledR}$ Version 2019-International Classification for Nursing Practice. International Council of Nurses; 2019. https://www.icn.ch/what-we-do/projects/ehealth-icnptm/icnp-browser. Accessed April 2021

11. Meleis A, Sawyer L, Im E, Messias D, Schumacher K. Experiencing transitions: an emerging middle-range theory. Adv Nur Sci. 2000; 23(1):12-28. https://doi.org/10.1097/00012272-200009000-00006

12. Meleis A. Transitions Theory Middle Range and Situation Specific Theories in Nursing Research and Practice. Springer Publishing Company; 2010.

13. Portuguese Nursing Council. Specific Competencies of the Mental Health Specialist Nurse. Portuguese Nursing Council (in Portuguese); 2010.

14. Portuguese Nursing Council. Advances to Improve Nursing Information Systems. 2020. https://www.ordemenfermeiros.pt/noticias/conteudos/ ordem-avan\%C3\%A7a-para-a-melhoria-dos-sistemas-de-informa\%C3\% A7\%C3\%A3o-em-enfermagem/. Accessed March 2021.

15. Krueger RA, Casey MA. Focus Groups: A Practical Guide for Applied Research. 5th ed. Sage; 2014.

16. Tong A, Sainsbury P, Craig J. Consolidated criteria for reporting qualitative research (COREQ): a 32-item checklist for interviews and focus groups. Int J Qual Health Care. 2007;19(6):349-357. https://doi.org/10. 1093/intqhc/mzm042

17. World Medical Association. World Medical Association Declaration of Helsinki: ethical principles for medical research involving human subjects. JAMA. 2013;310(20):2191-2194. https://doi.org/10.1001/jama. 2013.281053

18. Silva Abel. Nursing Informatics Systems, an Explanatory Theory of Change. Coimbra: Formasau, Training and Health Ltd.; 2006:

19. Buccheri R, Trygstad L, Buffum M, Birmingham P, Dowling G. Selfmanagement of unpleasant auditory hallucinations: a tested practice model. J Psychosoc Nurs Ment Health Serv. 2013;51(11):26-34. https://doi. org/10.3928/02793695-20130731-02

20. Buffum M, Buccheri R, Trygstad L, et al. Behavioral management of auditory hallucinations: implementation and evaluation of a 10-week course. J Psychosoc Nurs Ment Health Serv. 2009;47(9):32-40. https://doi. org/10.3928/02793695-20090730-0

21. Watson J. Clarifying the discipline of nursing as foundational to development of professional nursing. Editorial. Texto Contexto Enfermagem. 2017;26(4):1-2. https://doi.org/10.1590/0104-07072017002017edito rial4

22. Liu $\mathrm{Y}$, Tang $\mathrm{C}$, Hung $\mathrm{T}$, Tsai $\mathrm{P}$, Lin $\mathrm{M}$. The efficacy of metacognitive training for delusions in clients with Schizophrenia: a meta-analysis of randomized controlled trials Informs evidence-based practice. Worldviews Evidence-Based Nur. 2018;15(2):130-139. https://doi.org/10. 1111/wvn.12282

23. Moritz S, Woodward TS. Metacognitive training in schizophrenia: from basic research to knowledge translation and intervention. Curr Opin Psychiatry. 2007;20:619-625. https://doi.org/10.1097/YCO.0b013e328 2fob8ed

24. Ma YC, Beckstead JW, Lo SC, Yang CY. Auditory hallucinatory beliefs in clients with Schizophrenia: association of auditory hallucinations with social interactions, characteristics and emotional behaviors over 3 months. Arch Psychiatr Nurs. 2016;30(3):363-369. https://doi.org/10. 1016/j.apnu.2015.12.010

25. Erawati E, Keliat BA, Helena N, Hamid A. The influence of metacognitive training on delusion severity and metacognitive ability in schizophrenia. J Psychiatr Ment Health Nurs. 2014;21:841-847. https://doi.org/10.1111/ jpm.12130

26. Chadwick C, Hemingway S. A review of the effectiveness of interventions aimed at understanding the content and meaning of the experience of voice hearing. Ment Health Nurs. 2017;37(Issue 6):8-14.

27. Yang C, Lee T, Lo S, Beckstead JW. The effects of auditory hallucination symptom management programme for people with schizophrenia: a quasi-experimental design. J Adv Nurs. 2015;71(12):2886-2897. https:// doi.org/10.1111/jan.12754

28. Healthcare Information and Management Systems Society. Interoperability in healthcare. 2021. https://www.himss.org/resources/intero perability-healthcare. Aaccessed March 2021.

29. Gavrilov G, Vlahu-Gjorgievska E, Trajkovik V. Healthcare data warehouse system supporting cross-border interoperability. Health Informatics J. 2019;26(2):1321-1332. https://doi.org/10.1177/146045 8219876793

How to cite this article: Gonçalves PDB, Sequeira CAC, Paiva e Silva MATC, Paiva e Silva AA. Developing nursing clinical data models addressing delusion and hallucination with Meleis transitions theory as the theoretical reference model: A focus group study. Perspect Psychiatr Care. 2021;1-9. https://doi.org/10.1111/ppc.12869 